\title{
Editorial
}

\section{Advanced Nanomaterials for Energy-Related Applications}

\author{
Jiayu Wan, ${ }^{1}$ Taeseup Song, ${ }^{2}$ Cristina Flox, ${ }^{3}$ Junyou Yang, \\ Quan-Hong Yang, ${ }^{5,6}$ and Xiaogang Han ${ }^{1}$
}

\author{
${ }^{1}$ Department of Materials Science and Engineering, University of Maryland, College Park, MD 20742, USA \\ ${ }^{2}$ School of Materials Science and Engineering, Yeungnam University, Gyeongsan 712-749, Republic of Korea \\ ${ }^{3}$ Catalonia Institute for Energy Research, Jardins de les Dones de Negre, e1, Sant Adrià de Besòs, 08930 Barcelona, Spain \\ ${ }^{4}$ State Key Laboratory of Material Processing and Die \& Mould Technology, Huazhong University of Science and Technology, \\ Wuhan 430074, China \\ ${ }^{5}$ School of Chemical Engineering and Technology, Tianjin University, Tianjin 300072, China \\ ${ }^{6}$ Engineering Laboratory for Functionalized Carbon Materials, Graduate School at Shenzhen, Tsinghua University, \\ Shenzhen 518055, China
}

Correspondence should be addressed to Xiaogang Han; hanxg@umd.edu

Received 7 September 2015; Accepted 14 September 2015

Copyright (C) 2015 Jiayu Wan et al. This is an open access article distributed under the Creative Commons Attribution License, which permits unrestricted use, distribution, and reproduction in any medium, provided the original work is properly cited.

Energy has been the cornerstone of sustainable development of our economy and society. The dramatic demand on energy supply to be as unrestricted as possible, however, leads to conflicting feedback loops on the ecological environment. Thus, research is appealing worldwide to diminish the negative environmental consequences of energy consumption. This includes energy saving, generation, harvest, conversion, and storage. Nanomaterials and nanostructures provide unique mechanical, electrical, and optical properties and have played an important role in recent advances in energy-related applications.

In this special issue, we report the current progress on the preparation and the usage of nanomaterials for energyrelated applications. In these aspects, we will cover a broad range of subjects from nanomaterial synthesis and characterization to energy-related demonstration and relevant technologies and device. Specifically, we focused on the synthesis of nanomaterials and their application in Li-ion batteries and optoelectronic devices.

(1) Nanomaterials for Optoelectronic/Photochemical Devices. World energy consumption largely relies on the combustion of fossil fuels. However, the traditional way is neither sustainable nor environmentally benign. Renewable energy generation devices, such as solar cells, thermoelectronic devices, are widely investigated to replace fossil fuels. Nanomaterials have played an essential role in developing novel energy generation devices. The unusual quantum effect at nanoscale benefits the electron transport and band engineering in nanomaterials, which brings about an excellent performance for devices $[1,2]$.

In this special issue, we specifically focused on the nanomaterials/nanostructures for optoelectronic/photochemical devices. The papers "Graphene-Tapered $\mathrm{ZnO}$ Nanorods Array as a Flexible Antireflection Layer" and "Fabrication of an Antireflective Nanodome Array with High Packing Density for Photovoltaic Applications" reported the utilization of $\mathrm{ZnO} /$ graphene nanocomposite and nanodome structure as antireflection layer for solar cells, respectively. The nanofeature reduced the Fresnel reflection and increased the transmitted light through the solar cell. The paper "Enhanced Photovoltaic Properties of the Solar Cells Based on Cosensitization of $\mathrm{CdS}$ and Hydrogenation" reported the $\mathrm{CdS}$ quantum dots sensitized $\mathrm{TiO}_{2}$ porous nanocrystal with enhanced photovoltaic performance. The paper "Synthesis and Structural Characterization of $\mathrm{Al}_{2} \mathrm{O}_{3}$-Coated $\mathrm{MoS}_{2}$ Spheres for Photocatalysis Applications" reports the photocatalytic behavior of $\mathrm{Al}_{2} \mathrm{O}_{3}$ decorated $\mathrm{MoS}_{2}$ nanospheres; the enhanced performance of nanostructured $\mathrm{Al}_{2} \mathrm{O}_{3} / \mathrm{MoS}_{2}$ 
to bulk composite indicates it is a promising candidate for removal of pollutant from waste water.

(2) Nanomaterials for Energy Storage. Renewable energy storage has been ubiquitously utilized in the modernized world, such as portable electronics, electrical vehicles, and grid scale energy storage. Li-ion batteries, which obtain the highest massive/volumetric energy density among all existing technologies, have attracted most attention both in academia and industry. Still, in order to pursue Li-ion batteries with higher energy density and power density, novel materials and structures with high specific capacity and high rate performance are needed.

For example, the specific capacity for $\mathrm{Si}$ anode is $4200 \mathrm{mAh} / \mathrm{g}, 10$ times larger than that of graphite $(376 \mathrm{mAh} / \mathrm{g})$. However, large amount of $\mathrm{Li}$ insertion in Si caused a $400 \%$ volume change, thus resulting in severe pulverization problem/unstable solid electrolyte interphase (SEI) formation during cycling for bulk Si as Li-ion battery anode. To solve this problem, $\mathrm{Si}$ in various nanostructures is utilized; the best performed one achieved stable cycling of more than 2000 cycles $[3,4]$. The other advantage of nanomaterials for energy storage devices is that they obtain a large specific surface area. Large surface area is good for the accessibility of electrolyte, which is beneficial to the fast charge/discharge for Li-ion batteries and ultracapacitors [5].

In this special issue, we reported the exciting results of nanomaterials for energy storage applications. In the paper "Enhancement of Electrochemical Stability about Silicon/Carbon Composite Anode Materials for Lithium Ion Batteries," W. Xiao et al. reported the Si/C composite as anode for Li-ion batteries. This composite delivers a high charge capacity of $791.7 \mathrm{mAh} / \mathrm{g}$ at a current density as high as $500 \mathrm{mAh} / \mathrm{g}$. In the paper "Cauliflower-Like $\mathrm{Co}_{3} \mathrm{O}_{4} /$ ThreeDimensional Graphene Composite for High Performance Supercapacitor Applications," H. Liu et al. reported a $3 \mathrm{D}$ $\mathrm{Co}_{3} \mathrm{O}_{4}$ /graphene nanocomposite. In this composite, $\mathrm{Co}_{3} \mathrm{O}_{4}$ is used as active capacitor electrode and graphene is served as conductive additive and mechanical support. This composite shows an excellent specific capacity of $863 \mathrm{~F} / \mathrm{g}$ in $6 \mathrm{M} \mathrm{KOH}$ at the rate of $1 \mathrm{mV} / \mathrm{s}$.

(3) Synthesis of Nanomaterials for Energy Applications. Materials synthesis is the basis of nanomaterial applications in energy fields. Thus, this special issue also collected work focusing on synthesis of nanomaterials including $\mathrm{Ni}-\mathrm{Co}-$ coated carbon nanotubes ("Preparation and Microwave Absorbing Properties of an Electroless Ni-Co Coating on Multiwall Carbon Nanotubes Using $\left[\mathrm{Ag}\left(\mathrm{NH}_{3}\right)_{2}\right]^{+}$as Activator"), carbon dots ("Preparation and Application of Fluorescent Carbon Dots"), $\mathrm{MoS}_{2}$ nanoflowers and nanosheets ("Synthesis and Characterization of Molybdenum Disulfide Nanoflowers and Nanosheets: Nanotribology"), sodium aluminum hydride ("Synthesis of Renewable Energy Materials, Sodium Aluminum Hydride by Grignard Reagent of $\mathrm{Al}$ "), and nanocomposite of $\mathrm{Al}_{2} \mathrm{O}_{3}$ /Antifrogen $\mathrm{N}$ ("Classical Behavior of Alumina $\left(\mathrm{Al}_{2} \mathrm{O}_{3}\right)$ Nanofluids in Antifrogen $\mathrm{N}$ with Experimental Evidence"). All these materials were demonstrated for various energy applications such as microwave adsorption ("Preparation and Microwave Absorbing Properties of an Electroless Ni-Co Coating on Multiwall Carbon Nanotubes Using $\left[\mathrm{Ag}\left(\mathrm{NH}_{3}\right)_{2}\right]^{+}$as Activator"), heat transfer ("Classical Behavior of Alumina $\left(\mathrm{Al}_{2} \mathrm{O}_{3}\right)$ Nanofluids in Antifrogen $\mathrm{N}$ with Experimental Evidence"), lubrication ("Synthesis and Characterization of Molybdenum Disulfide Nanoflowers and Nanosheets: Nanotribology"), and photocatalysis ("Classical Behavior of Alumina $\left(\mathrm{Al}_{2} \mathrm{O}_{3}\right)$ Nanofluids in Antifrogen $\mathrm{N}$ with Experimental Evidence"). The synthesis methods involved electroless deposition ("Preparation and Microwave Absorbing Properties of an Electroless Ni-Co Coating on Multiwall Carbon Nanotubes Using $\left[\mathrm{Ag}\left(\mathrm{NH}_{3}\right)_{2}\right]^{+}$ as Activator"), solvothermal reaction ("Synthesis and Characterization of Molybdenum Disulfide Nanoflowers and Nanosheets: Nanotribology"), and traditional solid chemical reaction ("Synthesis of Renewable Energy Materials, Sodium Aluminum Hydride by Grignard Reagent of Al”).

\section{Jiayu Wan Taeseup Song Cristina Flox Junyou Yang Quan-Hong Yang Xiaogang Han}

\section{References}

[1] M. Law, L. E. Greene, J. C. Johnson, R. Saykally, and P. Yang, "Nanowire dye-sensitized solar cells," Nature Materials, vol. 4, no. 6, pp. 455-459, 2005.

[2] W. Bao, J. Wan, X. Han et al., "Approaching the limits of transparency and conductivity in graphitic materials through lithium intercalation," Nature Communications, vol. 5, article 4224, 2014.

[3] H. Wu, G. Chan, J. W. Choi et al., "Stable cycling of double-walled silicon nanotube battery anodes through solidelectrolyte interphase control," Nature Nanotechnology, vol. 7, no. 5, pp. 310-315, 2012.

[4] J. Wan, A. F. Kaplan, J. Zheng et al., "Two dimensional silicon nanowalls for lithium ion batteries," Journal of Materials Chemistry A, vol. 2, no. 17, pp. 6051-6057, 2014.

[5] P. Simon and Y. Gogotsi, "Materials for electrochemical capacitors," Nature Materials, vol. 7, no. 11, pp. 845-854, 2008. 

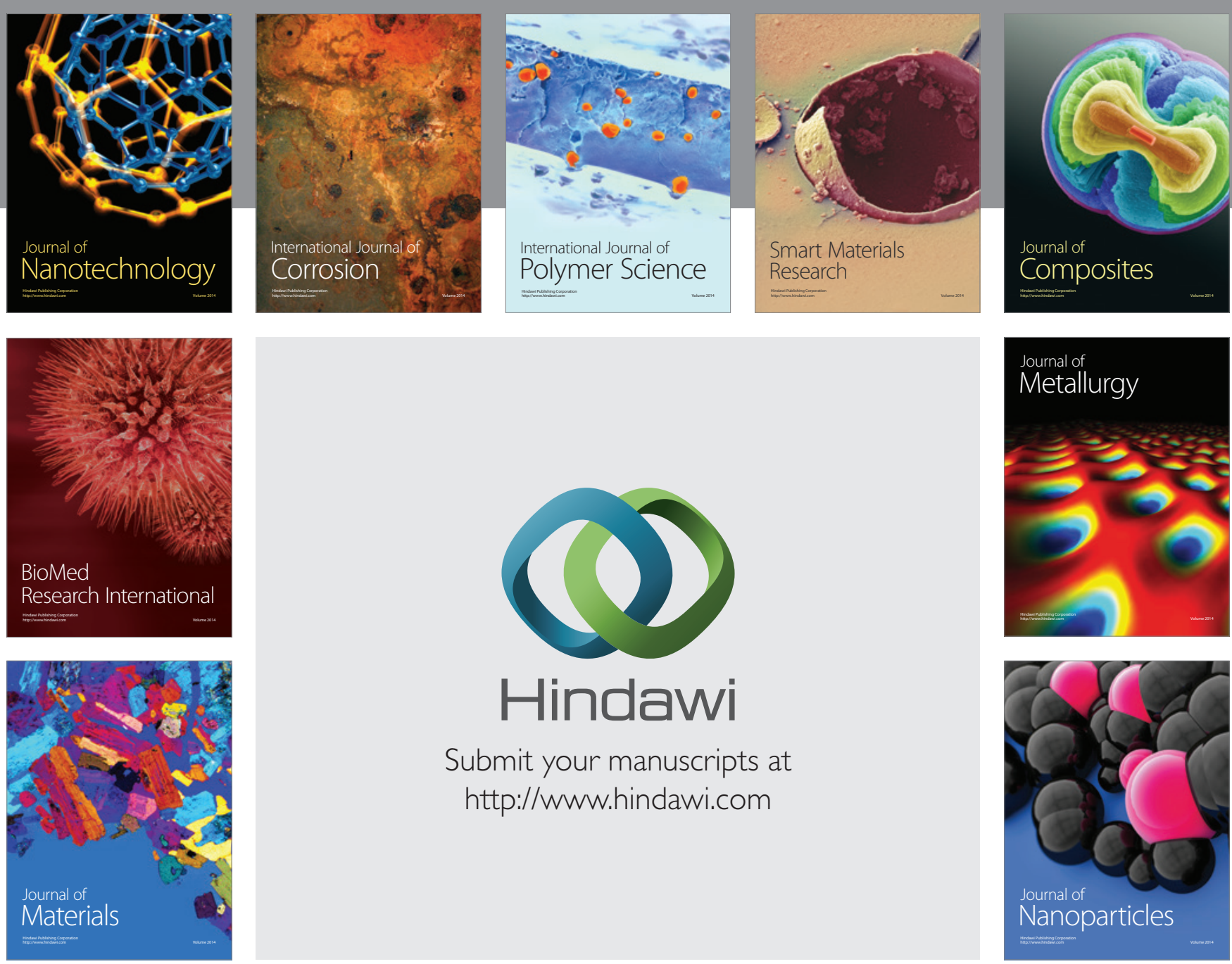

Submit your manuscripts at http://www.hindawi.com
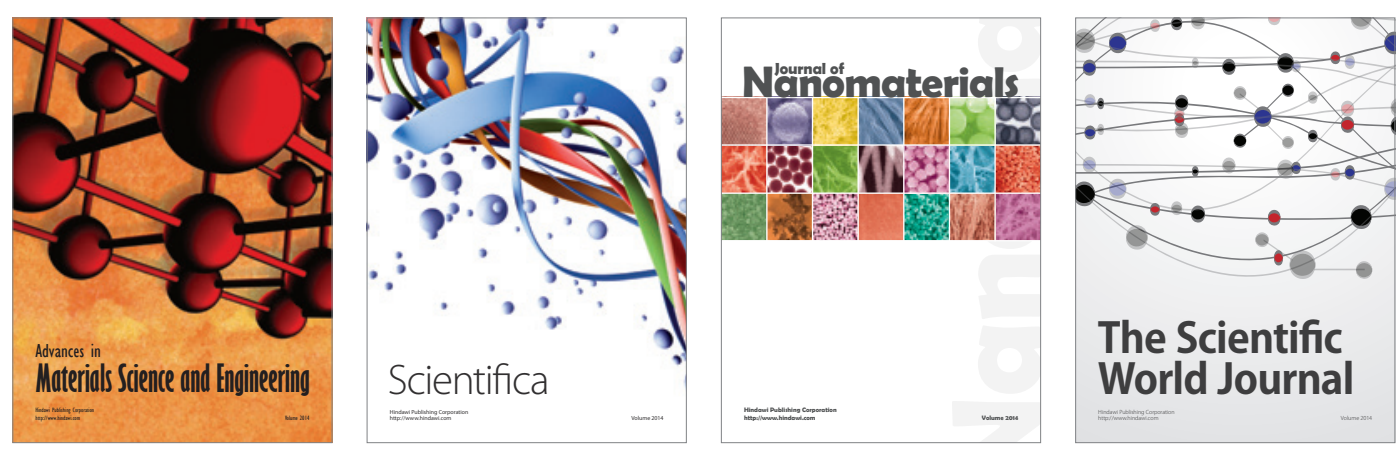

\section{The Scientific World Journal}
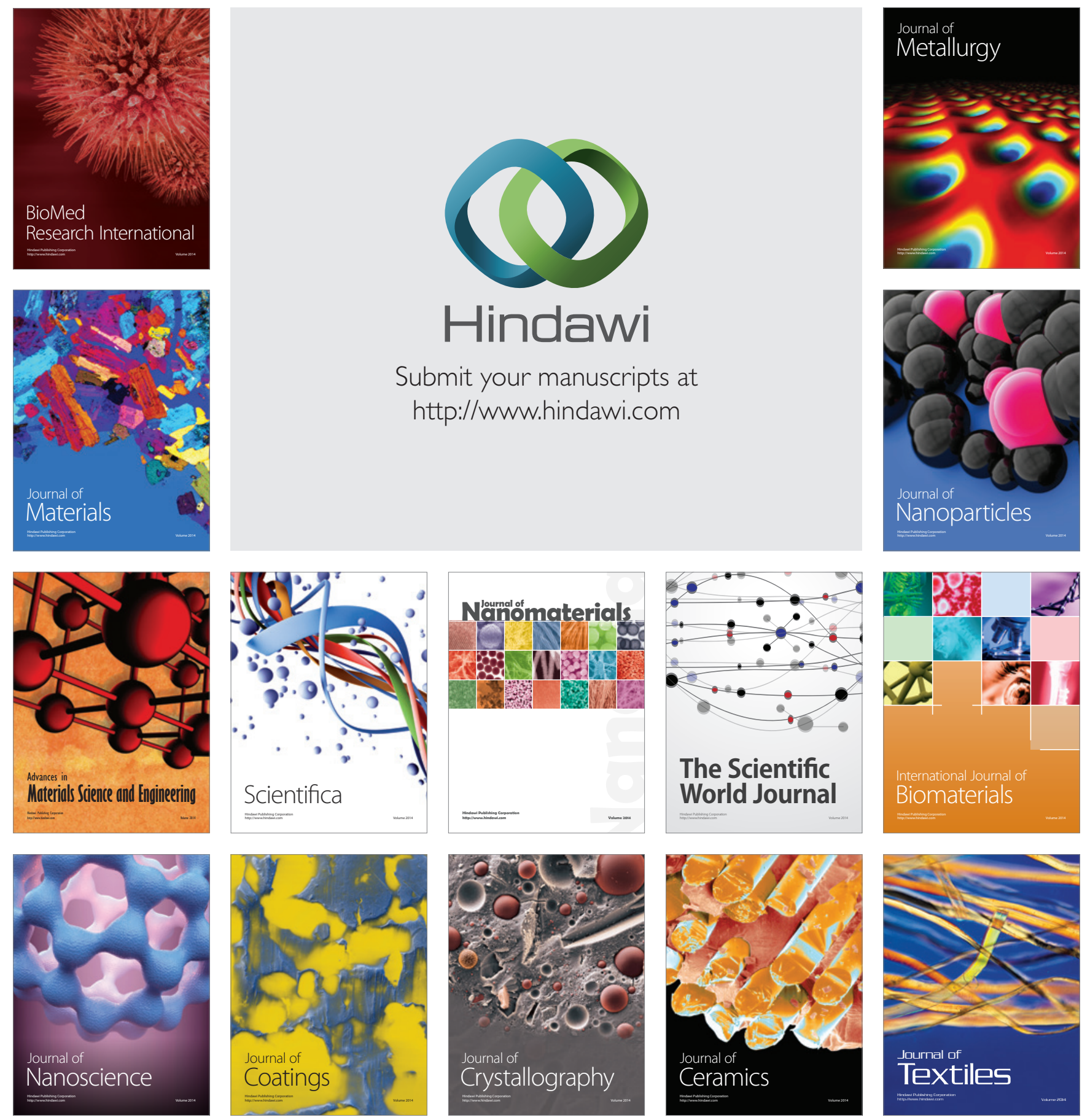